\title{
An Erratic Origin of Quadratus Plantae: An Embryological Error
}

\section{Sobana Mariappan ${ }^{1}$, Geeta Anasuya. D ${ }^{2}$, Sheela Grace Jeevamani MS ${ }^{3}$,} M. Vijaianand MD 4 .

${ }^{1}$ Tutor, Department of Anatomy, KMCH Institute of Health Sciences and Research, Coimbatore, India.

2 Associate Professor, Department of Anatomy, KMCH Institute of Health Sciences and Research, Coimbatore, India.

${ }^{3}$ Professor \& Head, Department of Anatomy, KMCH Institute of Health Sciences and Research, Coimbatore, India.

${ }^{4}$ Associate Professor, Department of Anatomy, $\mathrm{KMCH}$ Institute of Health Sciences and Research, Coimbatore, India.

\section{ABSTRACT}

Background: Quadratus plantae (Flexor digitorum accessorius) is one of the plantar muscles of foot . It is present in the second layer of sole. It takes origin from calcaneus and gets inserted into the tendon of flexor digitorum longus. The main function of it is to flex the lateral four toes in any position of the ankle joint by pulling on tendons of the flexor digitorum longus. Its variations like high origin have been implicated in the causation of tarsal tunnel syndrome.

Methodology and Results: In routine dissection done on 22 cadavers, we observed a bilateral variant muscle flexor digitorum accessorius longus on both right and left sides in a male cadaver. The modality of choice in diagnosing the accessory muscle is magnetic resonance imaging.

Conclusion: The knowledge of this variation would be essential to anatomists, radiologists and also to the foot surgeons while performing posterior ankle endoscopy.

KEY WORDS: Flexor digitorum Accessorius longus, Tarsal tunnel syndrome, Posterior ankle endoscopy.

Corresponding Author: Dr Geeta Anasuya MD, Associate Professor, Dept of Anatomy, $\mathrm{KMCH}$ Institute of Health Sciences \& Research, Coimbatore- 641014, Tamil Nadu, India.

E-Mail: geetagee1@gmail.com

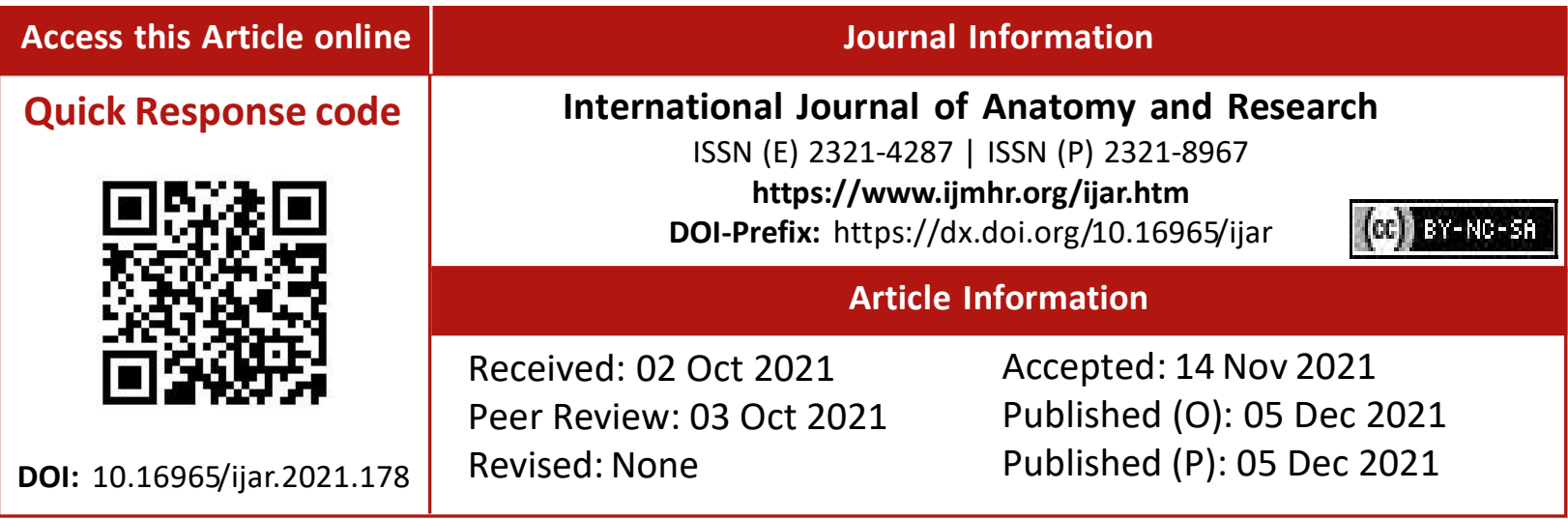

\section{INTRODUCTION}

Supernumerary muscles are most commonly the inherited ailments involving the growth of an extra portion of the body and an aberration from the body design. Supernumerary muscles in the leg and foot could be a factor for compression of tibial nerve in tarsal tunnel syndrome. Various supernumerary and variant muscles have been described in radiological, anatomical and surgical studies.

In the leg, posterior compartment is the largest and it gradually reduces as it reaches the ankle. The flexor digitorum longus is a thin, pointed muscle proximally and gradually widens distally. It arises from the posterior surface of tibia medial to tibialis posterior from just below the soleal line to within 7 or $8 \mathrm{~cm}$ of the distal end of bone. It also arises from 
the fascia covering the tibialis posterior. The muscle ends in tendon along whole of its posterior surface. Later, passes deep to flexor retinaculum and enters sole of foot. In the sole, it forms whole of long flexor tendons for second to fifth toes. The long flexor tendons of the lateral four toes are attached to the plantar surfaces of the bases of their distal phalanges.

The tendons of quadratus plantae or flexor digitorum accessorius insert into the flexor digitorum longus tendons of the second, third and fourth toes [1]. The normal insertion of quadratus plantae has been shown in Fig.1\&1a. Flexor digitorum accessorius arises by two heads with the long plantar ligament situated deeply in the interval between the two heads. It gets inserted into the tendon of flexor digitorum longus where it is bound by a fibrous slip to the tendon of flexor hallucis longus and here it divides into four tendons [2]. Accessory muscles around the ankle include flexor digitorum accessorius longus, peroneo calcaneus internus, the accessory soleus, accessory peroneal muscles[2].

Awareness of these accessory muscles existence and as one of the factors for compression in tarsal tunnel syndrome is essential [3].

\section{CASE REPORT}

During the routine dissection on 44 lower extremities of 22 embalmed cadavers, of both sexes for undergraduate students in the department of Anatomy, Karpagam faculty of medical sciences and research, we observed an erratic variation of flexor digitorum accessorius longus muscle in one male cadaver. Dissection of the lower extremities was done as per the steps provided by the Cunningham's manual of Practical Anatomy. The posterior compartment of leg has been dissected deep to soleus to reach the long flexors of toes. Later these tendons were traced distally till flexor retinaculum. Incision was extended to the sole, divided the plantar aponeurosis distal to heel to expose the three muscles of first layer of sole. To approach the second layer, the flexor digitorum brevis was cut in the middle and reflected the distal part distally to observe the second layer muscles [4].

Variant muscles were observed in both right and left lower limbs in a male cadaver. On left side, the origin of flexor digitorum accessorius longus muscle was aponeurotic and from the medial border of shaft of tibia above the soleal line proximal to origin of soleus muscle. The muscle belly was well defined and bipennate, and is inserted as a tendon to the juncture of flexor digitorum longus and flexor digitorum accessorius (Fig. 3 \& 3a). On the right side, the origin of flexor digitorum accessorius longus was same as left side. But the insertion was tendinous and passed deep to the flexor retinaculum and reached the flexor digitorum accessorius (Fig. 2 $\& 2 a$ ). Measurements of the muscle ( fleshy part, aponeurotic part and tendon part) was done using digital callipers (Table 1).

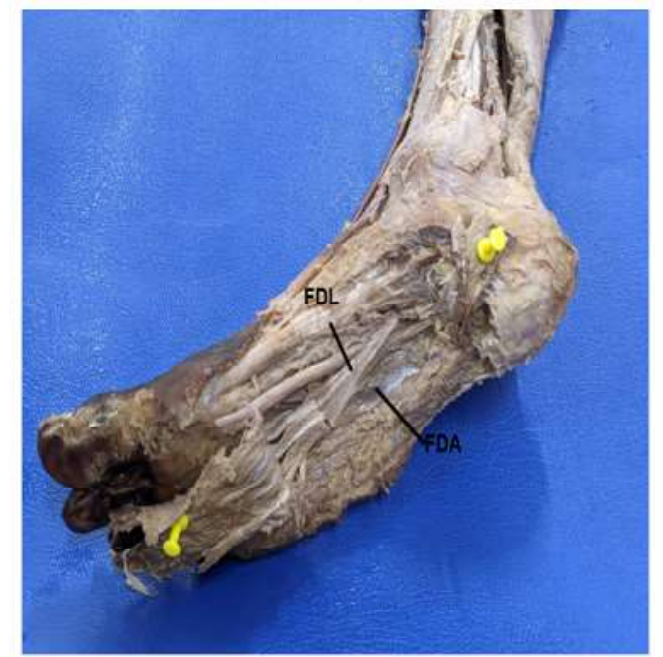

Fig. 1: Showing flexor digitorum longus (FDL) and Flexor digitorum accessories (FDA) on right foot.

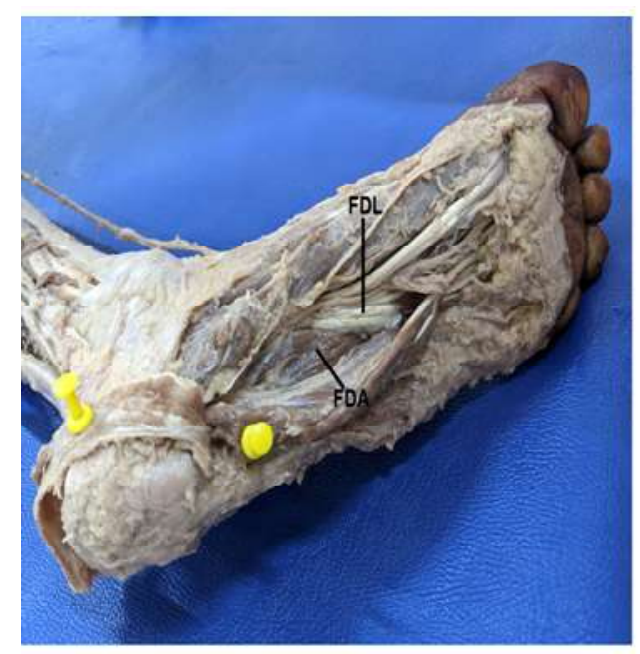

Fig. 1a: Showing flexor digitorum longus (FDL) and Flexor digitorum accessories (FDA) on left foot. 




Fig. 2: Showing a small muscle FDAL arising from medial border of shaft of right tibia Flexor digitorum accessorius longus (FDAL).

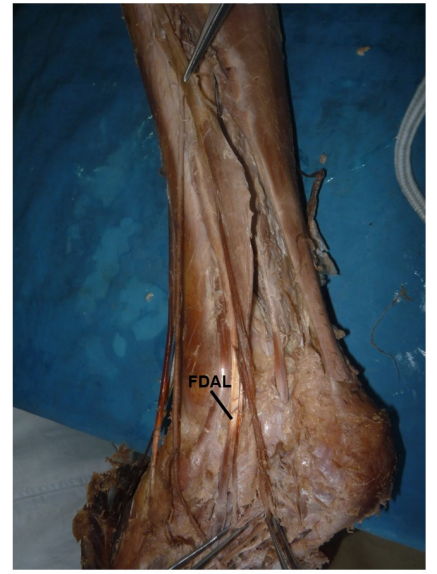

Fig. 3: Showing a small muscle FDAL arising from medial border of shaft of the left tibia.

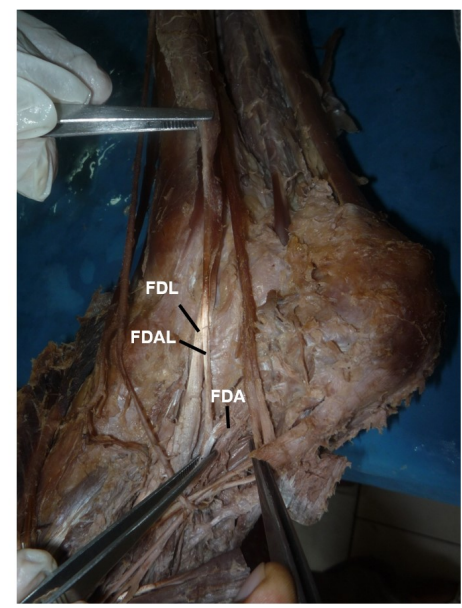

Fig. 2a: Showing the insertion of FDAL to FDA before joining with FDL on right side.

FDA- Flexor Digitorum accessorius, FDL- Flexor digitorum longus.

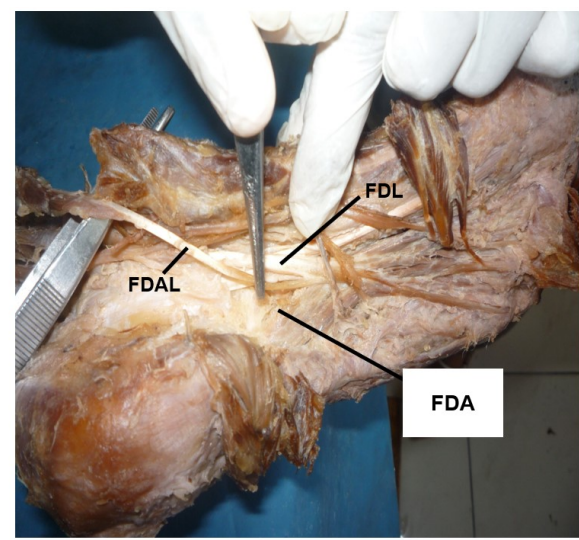

Fig. 3a: Showing the insertion to FDAL at the juncture of FDL and FDA on left side.

Table 1: Showing measurements of flexor digitorum accessorius longus (FDAL) on both right and left sides.

\begin{tabular}{cccccc}
\hline S.No & $\begin{array}{c}\text { Side of the } \\
\text { muscle }\end{array}$ & Upper part of FDAL & $\begin{array}{c}\text { Middle part of } \\
\text { FDAL }\end{array}$ & $\begin{array}{c}\text { Lower part of } \\
\text { FDAL }\end{array}$ & $\begin{array}{c}\text { Total length of } \\
\text { FDAL }\end{array}$ \\
\hline $\mathbf{1}$ & Right side & Aponeurotic $-5 \mathrm{~cm}$ & Fleshy $2 \mathrm{~cm}$ & Tendon $3 \mathrm{~cm}$ & $10 \mathrm{~cm}$ \\
$\mathbf{2}$ & Left side & Aponeurotic $-4 \mathrm{~cm}$ & Fleshy $1 \mathrm{~cm}$ & Tendon $3.5 \mathrm{~cm}$ & $8.5 \mathrm{~cm}$ \\
\hline
\end{tabular}

\section{DISCUSSION}

The erratic variation of flexor digitorum accessorius longus can be correlated with embryonic development. Right and left limbs of an embryo are pertinent to develop as mirror image so symmetry being expected as "a priori" [4]. Here, the variation was found to be bilateral.

The growth of a muscular mass into various special new muscles could be through various means. The original muscle may split into proximal and distal parts. The effect of such division into proximal and distal parts was very useful for reptiles, as they act independently of each other and also bring specialised actions. Long flexors and tensors of toes represent the distal parts of muscles taking origin from trunk and extending upto the toes. In amphibians, we could observe origin of short flexors from tendons of long flexors but it is an exception in mammals [5]. The flexor digitorum accessorius longus is a variant muscle with a reported prevalence of 2 to $12 \%$ in cadaveric studies, and $6 \%$ of asymptomatic individuals on MRI. Detecting the accessory muscle would prevent the muscle structure lesions and they have to be taken into consideration during posterior ankle arthroscopy [6]. 
An extra head of muscle, flexor accessorius longus with its tendon might originate from fibula, tibia or deep fascia and get inserted to the flexor digitorum longus or into quadratus plantae. Few small slips may extend to tibialis anterior or to flexor hallucis longus [1]. It was noticed that there was bilateral flexor digitorum accessorius longus in a male cadaver in the present case. A small muscle originated as aponeurosis from the medial border of shaft of tibia, middle part was fleshy and got inserted as tendon to flexor digitorum accessorius before joining with flexor digitorum longus on right side. On left side, similar slip like a mirror image originated from the medial border of tibia, middle part was fleshy and got inserted as tendon to the juncture of flexor digitorum accessorius and flexor digitorum longus.

Various types of accessory muscles in the foot and ankle have been described. Best described tendon of fourth fibularis in the posterolateral region, tendons of fibulo calcaneal ligament in the posteromedial region, long flexor accessory flexor of the toes, internal tibiocalcaneal ligament and accessory soleus [7]. However many times they do not cause any symptoms, but in athletes due to disproportionate loading they can result in pain, instability and block in joint. They can also lead to tarsal tunnel syndrome, hallux flexor syndrome and sprains $[7,8]$.

High origin of this muscle from tibia and fibula was observed in higher mammals, but in humans it was observed in sole [9].

Few studies observed its higher level of origin from the lower third of fibula and also from the fascia covering the flexor hallucis longus [10]. Jaijesh et al described its origin from the popliteal origin [11]. Athavale et al have reported about the origin as head of flexor digitorum accessorius and the presence of flexor digitorum accessories longus in two out of 47 cadavers [12]. The method of choice for diagnosing the accessory muscles is Magnetic resonance imaging (MRI). These muscles are isotense to skeletal muscles on all pulse sequences. During the execution of double incision compartment fasciotomy, the difference in the anatomical relationships along the medial side of leg must be noted by surgeons in order to obtain a complete release of the muscular portion of the deep posterior compartment of leg [13].

In addition to anatomical compression of neurovascular structures, injury can occur iatrogenically to tibial nerve during fasciotomy, tibial osteotomies and while harvesting the fibular graft [14].

\section{CONCLUSION}

The knowledge of accessory muscles is very essential as they could be confused with soft tissue lesions. Compression of the nerves by accessory muscles can occur anatomically and iatrogenic injury can also occur. These observations of the present study may be useful for radiologists for correct diagnosis as well as for surgeons in foot and ankle interventions.

\section{Conflicts of Interests: None}

\section{REFERENCES}

[1]. Strandring. S. Gray'sAnatomy : The Anatomical Basis of Clinical Practice $41^{\text {st }}$ edition. Newyork : Churchill Livingstone 2008; 1410- 42.

[2]. Carrol JF. Accessory muscles of the ankle. MRI web clinic - November 2008

[3]. Nathan H, Gloobe H, Yosipovitch Z. Flexor digitorum accessorius longus. Clin Orthop Relat Res.1975;113:158-161.

[4]. Keen JA. A study of the arterial variations in the limbs, with special reference to symmetry of vascular pattern. Am J A nat. 1961;108:245-261.

[5]. Gadow H. Observations in comparative myology . J Anat Physiol. 1882; 16:493-514.

[6]. Jorge Pablo Batista , Jorge Javier del Vecchio, Pau Golano, Jordi Vega. Flexor Digitorum Accessorius Longus: Importance of Posterior Ankle Endoscopy . 2015; 015: 823107

[7]. Best A, Giza E, Linnklater J, Sullivan M. Posterior impingement of the ankle caused by anomalous muscles. J Bone Joint Surg Am . 2005;87 (9): 2075-9.

[8]. Kinoshita M, Okuda R, Morikawa J, Abe M. Tarsal tunnel associated with an accessory muscle. Foot Ankle Int.2003;24(2):132-6.

[9]. De canter, K J Siesel. Flexor digitorum accessorius longus muscle : an etiology of tarsal tunnel syndrome ? 1997;36(3):226-9.

[10]. Wood J. Variations in human myology observed during the winter session of 1867-68 at King's college, London. Proc

[11]. Jaijesh P, Shenoy M, Anuradha L, Chitralekha KK. Flexor accessorius longus: A rare variation of the deep extrinsic digital flexors of the leg and its phylogenetic significance. Indian J Plast Surg 2006;39:169-71. 
[12]. Athavale SA, Geetha GN, Swathi. Morphology of flexor digitorum accessories muscle. Surg Radiol Anat 2012; 34:367-72

[13]. Cheney RA, Melaragno PG, Prayson MJ, Bennett $\mathrm{GL}$, Njus GO. Anatomic investigation of the deep posterior compartment of the leg. Foot Ankle Int.1998;19: 98-101.
[14]. Nayak SB, Shetty SD. Two accessory muscles of leg: potential source of entrapment of posterior tibial vessels. Surg Radiol Anat. 2019 Jan;41 (1): 97-99.

How to cite this article:

Sobana Mariappan, Geeta Anasuya. D, Sheela Grace Jeevamani MS, M. Vijaianand MD. An Erratic Origin of Quadratus Plantae: An Embryological Error. Int J Anat Res 2021;9(4):8168-8172. DOI: 10.16965/ijar.2021.178 